\title{
Worlds and models in Bayart and Carnap
}

\author{
M.J. Cresswell \\ Victoria University of Wellington \\ max.cresswell@vuw.ac.nz
}

\begin{abstract}
In the early days of the semantics for modal logic the 'possible worlds' were thought of as models or interpretations. This was particularly so when the interpretation was of logical necessity or possibility, where this was understood in terms of validity. Arnould Bayart in 1958 may have been the first modal logician to argue explicitly against the identification of necessity and validity. This note contrasts his semantics with that provided by Rudolf Carnap in 1946, and examines Bayart's proof that if you identify necessity with validity then certain theorems of S5 are not valid. The proof is then examined using Carnap's semantics.
\end{abstract}

In early work on the semantics of modal logic we find that what have come to be called 'possible worlds' were assumed to be models. ${ }^{1}$ It is well known that Kripke [11] pointed out that the indices could be anything at all. ${ }^{2}$ What is less well known is that in the work of Arnould Bayart in the late 1950s Bayart explicitly repudiates the view that worlds are models. Bayart [1] begins $^{3}$ :

0 . To formulate a semantic theory of modal logic it is not sufficient to define for example, the necessary as that which is true in

\footnotetext{
${ }^{1}$ In particular see [6], [9] and [10]; though the work that I conjecture Bayart had in mind is probably Carnap's [2]. (Like Carnap, Bayart is only concerned with S5, and shews no awareness of the relational semantics developed by these other authors.)

${ }^{2}$ On p. 69 of [11], Kripke notes that in his 1959 paper [10] it would not be possible to have two distinct worlds in which all formulae have exactly the same truth values.

${ }^{3} \mathrm{I}$ am using the translation in [5]. That translation uses a terminology and notation which are more accessible than Bayart's own. The differences are explained in [5]. This note is concerned only with first-order modal logic (the Lower Predicate Calculus). Bayart defines validity and proves soundness for second-order modal logic as well. In a second paper-also translated in [5]-Bayart proves the completeness of first-order S5, and what he calls the 'quasi-completeness' of second-order S5.
}

Australasian Journal of Logic (13:1) 2016, Article no. 1 
every model and the possible as that which is true in some model. These definitions would do no more than introduce the notions of 'necessary' and 'possible' in the metalanguage. A semantics of modal logic demands that we assume an object language containing modal symbols and that we define under what conditions to attribute the values 'true' or 'false' to the formulae of this object language.

One can then very easily define the validity and satisfiability of formulae in this language and shew the soundness of such and such a deductive system, this soundness consisting in the fact that all derivable formulae in the considered systems are valid.

It is a theory of this kind which we propose to develop in the present article, inspired by the Leibnizian definition of necessity as truth in all possible worlds.

It is not, in our opinion, the task of the logician to examine the value of this Leibnizian metaphysics. We can confine ourselves to shewing that if one takes this metaphysics one can formulate for the modal logic S5 a semantical theory analogous to the formal semantic theories of non-modal logic.

The modal semantic theory leads us to consider relations of a special kind. These are relations whose extension varies in one world or another, and we give them the name of 'intensional relations'.

1. Letting $\mathrm{D}$ and $\mathrm{W}$ be two non-empty sets, not having any common elements, call D the 'set of individuals' and $W$ the 'set of worlds'. We say that these sets $\mathrm{D}$ and $\mathrm{W}$ constitute a universe $\langle\mathrm{D}, \mathrm{W}\rangle$, For each natural number $\mathrm{n}$ we mean by ' $\mathrm{n}$-place intensional relation' a function of $n+1$ arguments, taking the value $T$, 'true', or F, 'false', having a world as its first argument and for $\mathrm{n}$ $\neq 0$, having $\mathrm{n}$ individuals as its last $\mathrm{n}$ arguments.

At the close of that article Bayart gives examples to shew why you should not make the identification of worlds (or if you prefer, the more neutral 'indices') with models. If we use the symbol $\square$ for logical necessity, a model of the kind assumed by Bayart relativises truth to a set W of 'possible worlds' in the usual way, and the semantics for $\square$ can be provided by saying that a wff $\square \alpha$ is true in a model $\mathrm{M}$ at a world $w$ iff $\alpha$ is true at $w^{\prime}$ (in that model) for every $w^{\prime} \in \mathrm{W} .{ }^{4}$ With such a semantics, and provided you assume, as

\footnotetext{
${ }^{4}$ Bayart does not use the word 'model' here, though he has done so in paragraph 0. What he does is speak of is $<<$ un système de valeurs $S>>$, which I have translated as 'an interpretation $V^{\prime}$. Since both Bayart and Carnap use (different) notations which are not common today, and because of Bayart's use of $<<$ modèl $>>$ in paragraph 0 , I have used the
} 
Bayart does that there is just a single domain of individuals for every world, the class of valid formulae is the predicate system S5. (In which the Barcan Formula is a theorem.)

In section VI of [1], a section called 'Necessity and validity', Bayart presents his counterexample. ${ }^{5}$ He begins by telling us:

28. One might perhaps combine the notions of necessity and validity. One might then formulate the following semantic theory: Instead of providing a universe consisting of a domain and a set of individuals one might simply give a domain D, i.e., a set of individuals. One then gives a set of extensional relations. For each natural number $n$, an extensional relation is a function of $n$ arguments, these arguments being individuals, and able to take the values $T$ or $F$.

Individual variables can take individuals as values, and n-place predicate variables can take n-place extensional relations as values. Propositional variables can take T or F as values.

The motivation for this procedure is to articulate the claim that logical necessity should be interpreted so that $\square \alpha$ is true in a model iff $\alpha$ is valid, i.e. true in every model. As noted in footnote 4, Bayart does not use the word 'model' for his own semantics, and he does not do so here either, but it is clear that in Section VI he intends to consider interpretations which are not based on a set of worlds. For this purpose models are simply the ordinary sort of models for non-modal predicate logic. In order to avoid confusion with possible-worlds models, I will call such a model a model*. The one constraint that has to be imposed is that they are all based on the same set of individuals.

The kind of semantics that Bayart is about to criticise is plausibly attributed to Rudolf Carnap. Although Bayart includes [2] in his bibliography, he does

word 'model' in this note. Paragraph 3 begins:

3. Let $\langle\mathrm{D}, \mathrm{W}\rangle$ be a universe composed of the set $\mathrm{D}$ of individuals and $\mathrm{W}$ of worlds. We agree that the variables for individuals of the language $\mathcal{L}$ can take as values individuals of the set $\mathrm{D}$ and that for each natural number $\mathrm{n}$ the variables for $n$-place predicates take as values n-place intensional relations defined on the universe $\langle\mathrm{D}, \mathrm{W}\rangle$.

We take a universe $\langle\mathrm{D}, \mathrm{W}\rangle$, a world $w$ of this universe and an interpretation $\mathrm{V}$ relative to this universe. We then define the notions 'true for universe $\langle\mathrm{D}, \mathrm{W}\rangle$,

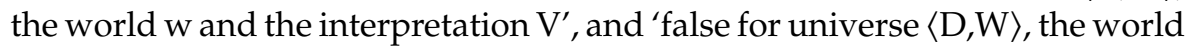
$w$ and the interpretation $\mathrm{V}^{\prime}$.

${ }^{5}$ Bayart actually presents two counterexamples, one for first-order logic and one for second-order logic.

Australasian Journal of Logic (13:1) 2016, Article no. 1 
not attribute 'validity' semantics to any particular author; but it is useful to proceed with Carnap in mind. I shall call the wff which are valid using this semantics *-valid. It is known (see for instance [4], p. 55) that this kind of validity is not axiomatisable. This is because, if it were, we could generate all the wff $\sim \square \alpha$ for which $\alpha$ is not a theorem, which would decide first-order logic. But that leaves open the possibility that although predicate S5 will not be complete for *-validity it might still be sound for *-validity-as it is in the propositional case ([3], p. 55). The purpose of this note is to discuss Bayart's proof that predicate S5 is not even sound with respect to ${ }^{*}$-validity. It is important here to point out that the mere treatment of a world as a model ${ }^{*}$ is not sufficient to give the 'validity' semantics. For instance Kripke 1959 [10] p. 2 defines a model as a set of 'complete assignments', each of which may be thought of as a model*. In [10], validity is defined as truth in every model-every set of complete assignments. To get the semantics Bayart has in mind you would have to allow just one set of complete assignmentsthe set consisting of every model*. Since Kripke allows a world to be an arbitrary set of complete assignments, his notion of validity is not the one that Bayart is presently criticising, and in fact Kripke's 1959 semantics is sound and complete for first-order S5.

After introducing the semantics described in paragraph 28 Bayart sets out a standard set of rules for the truth-functional operators. In interpreting the quantifiers we note that for Bayart an interpretation for predicate logic $\left(\mathrm{a}\right.$ model $^{*}$ ) provides the values of both the predicates and the individual variables $^{6}$ - Bayart has no individual constants. So his semantics for $\exists$ may be written, where $\mathrm{M}$ is any model* ${ }^{*}$, as follows:

(1) A wff of the form $\exists x \alpha$ is true in M iff $\alpha$ is true in some model* $\mathrm{M}^{\prime}$ which is just like $\mathrm{M}$ except for the value it gives to $x$

(with the corresponding semantics for $\forall$.) For the modal operators his rules, adapted to the present notation, are

(2) A wff of the form $\square \alpha$ is true in $\mathrm{M}$ if for every model ${ }^{*} \mathrm{M}^{\prime}, \alpha$ is true in $\mathrm{M}^{\prime}$, and otherwise it is false in $\mathrm{M}$.

(3) A wff of the form $\diamond \alpha$ is true in $\mathrm{M}$ if there is an model* $\mathrm{M}^{\prime}$, such that $\alpha$ is true in $\mathrm{M}^{\prime}$, and otherwise it is false in $\mathrm{M}$.

\section{After doing this he comments}

\footnotetext{
${ }^{6}$ Presumably this is because Bayart regards both predicates and individual variables as variables, a fact which is more realistic when you bear in mind that Bayart's papers also apply to second-order logic, where predicates can be bound by quantifiers. Bayart's procedure was adopted in Hughes and Cresswell 1968 [7], though not in Hughes and Cresswell 1996 [8], and is not very common today.
}

Australasian Journal of Logic (13:1) 2016, Article no. 1 
31. The semantic rules that we have just given make first-order S5 unsound, and equally in the second-order case.

Bayart then establishes that the formula $\exists y \square(\varphi x \vee \sim \varphi y)$ is a theorem of S5, but is not *-valid. The former is easy to shew using standard S5 proof methods (rather than Bayart's somewhat cumbersome derivations in Gentzen style):

$\mathrm{PC}$

(i) Necessitation

(ii) Existential generalisation

(iii) Universal generalisation (i) $\varphi x \vee \sim \varphi x$

(ii) $\square(\varphi x \vee \sim \varphi x)$

(iii) $\exists y \square(\varphi x \vee \sim \varphi y)$

(iv) $\forall x \exists y \square(\varphi x \vee \sim \varphi y)^{7}$

In order to prove that $\exists y \square(\varphi x \vee \sim \varphi y)$ is not valid in his semantics Bayart uses a model ${ }^{*}$ in which there are two individuals, 0 and 1 , and a predicate $\varphi$ which is true of 0 but false of 1 . Then, if $x$ is assigned 1 and $y$ is assigned $0, \varphi x \vee \sim \varphi y$ is false. By (2) this means that $\square(\varphi x \vee \sim \varphi y)$ is false in every model $^{*}$, so that $\exists y \square(\varphi x \vee \sim \varphi y)$, and therefore $\forall x \exists y \square(\varphi x \vee \sim \varphi y)$, is false in every model*.

Bayart diagnoses the problem in the concluding paragraph of his article:

33. The problem with the sematic theory presented in section VI lies in the fact that it treats the symbols $L$ and $M$ as abbreviations for universal and existential closures. So that in expressions of the form $\exists y L(\varphi x \vee \sim \varphi y)$ or $\exists \psi L(\varphi x \vee \sim \psi x)$ the variables $y$ and $\psi$ are considered to be bound by $L$ and $M$ and not by the quantifiers $\exists y$ or $\exists \psi$, as they are in modal logic. Modal logic does not treat $L$ and $M$ as abbreviations for universal or existential closure. In other words modal logic does not identify the notions of validity and necessity.

Carnap proceeds a little differently. In the first place he talks about statedescriptions, but it is not difficult to express his semantics for (first-order) non-modal logic in such a way that a state description can be understood as a model ${ }^{*}$. In the second place he represents the domain of the model ${ }^{*}$ by individual constants in the language, where each individual in the domain is represented by one and only one constant in the language. For Carnap, truth in a model ${ }^{*}$ is defined only for sentences-wff without free variables. Carnap's semantics for the existential quantifier, expressed in terms of model ${ }^{*}$, amounts to

\footnotetext{
${ }^{8}$ For more information on the technical details of Carnap's modal logic, see [3] for the propositional case, and [4] for the predicate case.
}

Australasian Journal of Logic (13:1) 2016, Article no. 1 
(4) $\exists x \alpha$ is true in M iff $\alpha[a / x]$ is true in M for some constant $a$. (Here $\alpha$ contains $x$ as its only free variable, and $\alpha[a / x]$ is $\alpha$ with $a$ replacing every free $x$. $)^{9}$

Since $\varphi a \vee \sim \varphi a$ is true, for every constant $a$ in every model ${ }^{*} \mathrm{M}^{\prime}$ we have

(5) $\square(\varphi a \vee \sim \varphi a)$ is true in $\mathrm{M}$

and so, by (4)

(6) $\exists y \square(\varphi a \vee \sim \varphi y)$ is true in $\mathrm{M}$.

Since (6) is true for every constant $a$ we have

(7) $\forall x \exists y \square(\varphi x \vee \sim \varphi y)$ is true in $\mathrm{M}$.

It is instructive to see where the difference between Carnap's semantics and Bayart's semantics arises. For Bayart, a model ${ }^{*}$ gives values to the individual variables, and therefore 'every model ${ }^{* \prime}$ includes all possible values for the individual variables. In current semantics for (first-order) predicate logic there is often a division of labour, since a model* or interpretation usually supplies values to the predicates, while the values to individual variables are supplied separately by an assignment of individuals from the domain. In what follows I assume a fixed infinite domain of individuals on which all the models are based. So suppose that $\mathrm{M}$ is a model ${ }^{*}$ and $\mu$ is an assignment from the domain of individuals to each individual variable. We can then say:

(8) $\exists x \alpha$ is true in $\mathrm{M}$ with respect to an assignment $\mu$ iff $\alpha$ is true in $\mathrm{M}$ with respect to some assignment $\rho$, where $\rho$ is just like $\mu$ except, possibly, for $\rho(x)$.

If we follow Bayart we can express the semantics for $\square$ as

(9) $\square \alpha$ is true in a model ${ }^{*} \mathrm{M}$ with respect to an assignment $\mu$ iff $\alpha$ is true in $\mathrm{M}^{\prime}$ where $\mathrm{M}^{\prime}$ is any model ${ }^{*}$, and $\rho$ is any assignment.

\footnotetext{
${ }^{9}$ Carnap himself does not speak of truth in a model, or even of truth in a state-description, but speaks of the range of a sentence, which is a set of state-descriptions. Where I say that a sentence is true in a model ${ }^{*}$, Carnap will say that a state-description is in the range of that sentence. Carnap's 'rules of ranges' ([2], p. 50) then become equivalent to what is set out in the present text.
}

Australasian Journal of Logic (13:1) 2016, Article no. 1 
Using (9) we have that $\square(\varphi x \vee \sim \varphi y)$ is false in every model* $\mathrm{M}$ for every assignment $\mu$, since it is easy to have a model ${ }^{*} \mathrm{M}^{\prime}$ with a predicate $\varphi$, and two individuals, 0 and 1 in Bayart's example such that 0 satisfies $\varphi$ while 1 does not, and this can be so even if $\mu(x)=\mu(y)=0$. In comparing an assignment semantics with a Carnapian semantics it is tempting to think of a Carnapian sentence like $\varphi a \vee \sim \varphi a$ as being what you get from $\varphi x \vee \sim \varphi x$, when the Carnapian constant $a$ is associated with 0 , and $\mu(x)=0$. But of course it can also be what you get from $\varphi x \vee \sim \varphi y$ when $\mu(x)=\mu(y)=0$. It is this flexibility which is traded on by Bayart, but is not available to Carnap.

The other way of interpreting $\square$ is to hold $\mu$ constant. The semantics then reads:

(10) $\square \alpha$ is true in a model ${ }^{*} \mathrm{M}$ with respect to an assignment $\mu$ iff $\alpha$ is true in $\mathrm{M}^{\prime}$ with respect to $\mu$ where $\mathrm{M}^{\prime}$ is any model ${ }^{*}$.

If we use (10), if $\mu(x)=\mu(y)$ then $\square(\varphi x \vee \sim \varphi y)$ will be true in every $\mathrm{M}^{\prime}$ with respect to $\mu$, and therefore, by (8),

(11) $\exists y \square(\varphi x \vee \sim \varphi y)$

will be true in every $\mathrm{M}^{\prime}$ for every assignment $\rho$, and so,

(12) $\forall x \exists y \square(\varphi x \vee \sim \varphi y)$

will be valid.

The assignment semantics enables you to make a choice between (9) and (10). By having the model ${ }^{*}$ give values to the individual variables as well as the predicate variables, Bayart's version of the validity semantics requires that (2) be understood as (9) while Carnap's semantics requires that (2) be understood as (10). The assignment semantics is pretty much the same as Tarski's notion of a formula's being satisfied by a sequence of individuals. Carnap's semantics is no longer widely used, except perhaps by those who espouse what is sometimes called the 'truth value semantics'. ${ }^{10}$

The distinction between values given by the model and values given by assignments applies easily to languages like Bayart's, in which there are no individual constants. Where individual constants occur, their values are normally given by the model in the same way as values are given to the predicates; and this means that we can keep (10), and argue as follows. Consider a model* $\mathrm{M}$ like the one provided by Bayart in which there are two

\footnotetext{
${ }^{10}$ The truth-value, or sometimes 'domainless', semantics is discussed in [13], but also lies behind the earlier [12]. More material on this view was subsequently published in [14].
}

Australasian Journal of Logic (13:1) 2016, Article no. 1 
individuals, 0 and 1 , and a predicate $\varphi$ which is true of 0 but false of 1 . Then, if $a$ is a constant whose value in the model is 1 , and $b$ is a constant whose value is $0, \varphi a \vee \sim \varphi b$ is false in $\mathrm{M}$. By (10) this means that

(13) $\square(\varphi a \vee \sim \varphi b)$ is false in every model $^{*} \mathrm{M}^{\prime}$.

And, since $\square(\varphi a \vee \sim \varphi b)$ contains no individual variables, (13) is true with respect to every assignment. Let $\rho$ be any assignment to the individual variables. Then there will be a model ${ }^{*} \mathrm{M}^{\prime}$ which is just like $\mathrm{M}$ except that $\mathrm{M}^{\prime}$ gives $b$ the value $\rho(y)$. So, by (13),

(14) $\square(\varphi a \vee \sim \varphi y)$ is false in $\mathrm{M}^{\prime}$ with respect to $\rho$.

Now $b$ does not occur in $\exists y \square(\varphi a \vee \sim \varphi y)$ and so

(15) $\square(\varphi a \vee \sim \varphi y)$ is false in $\mathrm{M}$ with respect to $\rho$.

Since this is so for every $\rho$, then by (8)

(16) $\exists y \square(\varphi a \vee \sim \varphi y)$ is false in $M$ with respect to every assignment $\mu$.

So, by the dual of (8) for $\forall$,

(17) $\forall x \exists y \square(\varphi x \vee \sim \varphi y)$ is false in $\mathrm{M}$ with respect to $\mu$.

In order for (10) to make a difference in a language with constants one would have to make it read

(18) $\square \alpha$ is true in a model ${ }^{*} \mathrm{M}$ with respect to an assignment $\mu$ iff $\alpha$ is true in $\mathrm{M}^{\prime}$ with respect to $\mu$, where $\mathrm{M}^{\prime}$ is any model ${ }^{*}$ which coincides with $\mathrm{M}$ in the values it gives to all individual constants.

If (18) is used in place of (10) there would then need to be some discussion about why logical necessity means truth no matter what the predicates mean, but not truth no matter what the individual constants mean.

If, like Bayart, you don't think of a world as a model* then there is no change of model as you move from world to world, and what you get is simply the now standard Kripke semantics: 
(19) $\square \alpha$ is true in a model $M$ with respect to an assignment $\mu$ in a world $w$ iff $\alpha$ is true in $\mathrm{M}$ with respect to $\mu$ for every world $w^{\prime}$.

The purpose of this note is not to argue for the superiority of Bayart's semantics over Carnap's, or vice versa. It is simply to note that the identification of worlds and models is trickier than one might suppose, and that it is therefore of some interest that Arnould Bayart saw the dangers at least as early as 1958, and may have been the first modal logician to publish a warning about them; and perhaps also the first to see that "worlds" in modal logic can be anything at all.

\section{References}

[1] Arnould Bayart. La correction de la logique modale du premier et second ordre S5. Logique et Analyse, 1:28-44, 1958.

[2] Rudolf Carnap. Modalities and quantification. The Journal of Symbolic Logic, 11:33-64, 1946.

[3] Max Cresswell. Carnap and McKinsey: Topics in the pre-history of possible worlds semantics. In R. Goldblatt J. Brendle, R. Downey and B. Kim, editors, Proceedings of the 12th Asian Logic Conference, pages 53-75. World Scientific, 2013.

[4] Max Cresswell. The completeness of Carnap's predicate logic. Australasian Journal of Logic, 11:47-63, 2014.

[5] Max Cresswell. Arnould Bayart's modal completeness theorems: translated with an introduction and commentary. Logique et Analyse, (229):89142, 2015.

[6] K. J. J. Hintikka. Quantifiers in Deontic Logic. Helsingfors, Societas Scientiarum Fennica. Commentationes Humanarum Litterarum, 23.4. 1957.

[7] G.E. Hughes and M.J. Cresswell. An Introduction to Modal Logic. Methuen, 1968.

[8] G.E. Hughes and M.J. Cresswell. A New Introduction to Modal Logic. Routledge, 1996.

[9] S. G. Kanger. Provability in Logic. Almqvist \& Wiksell, 1957. 
[10] Saul Kripke. A completeness theorem in modal logic. The Journal of Symbolic Logic, 24:1-14, 1959.

[11] Saul Kripke. Semantical analysis of modal logic I, normal propositional calculi. In Zeitschrift fr mathematische Logik und Grundlagen der Mathematik, volume 9, pages 67-96. 1963.

[12] H. Leblanc. A simplified account of validity and implication for quantificational logic. The Journal of Symbolic Logic, 33:231-235, 1968.

[13] H. Leblanc. Semantic deviations. In [14], pages 1-16. 1973.

[14] H. Leblanc. Truth, Syntax and Modality. North Holland, Amsterdam, 1973.

Australasian Journal of Logic (13:1) 2016, Article no. 1 